\title{
Simultaneous analysis of cytoskeletal patterns and chromosome positioning in human fertilization failures
}

\author{
João Ramalho-Santos, Ph.D., ${ }^{\mathrm{a}}$ Alexandra Amaral, ${ }^{\mathrm{a}}$ Raquel Brito, ${ }^{\mathrm{b}}$ \\ Mariana Freitas, M.Sc., ${ }^{\mathrm{a}}$ and Teresa Almeida Santos, M.D., Ph.D. ${ }^{\mathrm{b}}$ \\ Center for Neuroscience and Cell Biology, University of Coimbra; and University Hospitals of Coimbra, \\ Coimbra, Portugal
}

Objective: To sequentially and reliably apply both tubulin immunocytochemistry (ICC) and fluorescence in situ hybridization (FISH) to human fertilization failures, thus providing a tool for a multiple analysis of arrest.

Design: Analysis of human fertilization failures at several stages of arrest.

Setting: Academic and clinical institutions.

Patient(s): Consenting patients undergoing assisted reproduction technologies.

Intervention(s): Failed fertilizations displaying signs of activation without pronuclear development, or with the absence of polar body emission or cleavage 48 hours after insemination or microinjection were analyzed. Fertilization failures were fixed and processed for ICC. After data was collected the same samples were then subjected to FISH analysis using probes for chromosomes $\mathrm{X}, \mathrm{Y}$, and 18.

Main Outcome Measure(s): Simultaneous ICC and FISH data on the same sample.

Received January 7, 2004; revised and accepted May 4, 2004.

Supported by a grant from the Portuguese National Science Foundation (Fundação para a Ciência e Tecnologia, FCT) to J. R.-S. (POCTI/ESP/38049/2001).

A.A. is supported by a Ph.D. grant from FCT, Portugal (SFRH/BD/12665/2003).

Reprint requests: João Ramalho-Santos, Ph.D., Center for Neuroscience and Cell Biology, Department of Zoology, University of Coimbra, 3004-517 Coimbra, Portugal (FAX: + 351-239826798; E-mail: jramalho@ci.uc.pt).

${ }^{a}$ Center for Neuroscience and Cell Biology, Department of Zoology, University of Coimbra.

${ }^{\mathrm{b}}$ Department of MaternalFetal Medicine, Genetics and Human Reproduction, University Hospitals of Coimbra.

0015-0282/04/\$30.00 doi:10.1016/j.fertnstert.2004 05.086

Result(s): Sequential application of straightforward standard ICC and FISH techniques was not possible, as the morphologic features had been altered, microtubular patterns were not preserved, and many samples were rendered opaque. Only chromatin at the cell surface or outside the oocyte/zygote, such as metaphase II spindles or polar body nuclei, could be routinely probed for FISH after ICC. However, an increase in detergent-induced sample permeabilization as well as the removal of several steps usually performed for FISH made it possible to directly compare microtubular patterns and chromosome position, regardless of chromatin status.

Conclusion(s): Analysis of specific proteins by immunocytochemistry and of chromosome status/positioning by FISH can be carried out sequentially in human fertilization failures, irrespective of the stage of arrest. (Fertil Steril ${ }^{\circledR}$ 2004;82:1654-9. (C2004 by American Society for Reproductive Medicine.)

Key Words: Human fertilization failures, microtubules, FISH, human oocyte, immunocytochemistry

By definition, analysis of failed human fertilization takes place after the fact, by evaluating oocytes/zygotes that failed to develop properly after IVF or intracytoplasmic sperm injection (ICSI) or were otherwise deemed unsuitable for embryo transfer. This leads to several limitations, not least of which is the fact that appropriate controls (i.e., normally developing embryos) are rarely, if ever, available for analysis. On the other hand, technical constraints often imply that a sample that is analyzed using a particular approach is rendered useless for further procedures, which require different preparatory steps. This is particularly important for two reasons. First, the intrinsic preciousness of the material as well as the uniqueness of any given sample limits the number of statistically significant experiments that can be done in a given laboratory/clinical setting, especially one without access to relevant animal models. Second, given that a failure in fertilization probably depends on several factors (1), a multiparameter analysis, which would probe a single oocyte/zygote for several possible miscues, could be useful to determine the causes of failure as well as to aid in the separation of causes from consequences.

The use of tubulin immunofluorescence to investigate the status of the metaphase II spindle in human oocytes is well established (2). On the other hand, fluorescence in situ hybridization (FISH) has also been an important tool 
to analyze chromosome status in human oocytes/fertilization failures (3). The importance of proper microtubular assembly patterns during fertilization includes spindle formation during meiosis, but also extends to pronuclear apposition mediated by the sperm aster formed from the male-borne centrosome and to the subsequent organization of the embryo's mitotic apparatus $(4,5)$. Indeed, microtubular patterns have been used to pinpoint several stages of arrest in human failed fertilizations (2). Such an analysis would be potentiated if information for chromosome status and position were available for the same sample, that is, if following immunocytochemistry (ICC) the same oocyte/zygote could be submitted to FISH without the original structural configuration being affected.

The sequential use of ICC and FISH has been described, notably employing both DNA probes and antibodies against chromosome-associated proteins (6-9). This analysis has also been extended to the evaluation of oocyte status, work remarkably pioneered in the mouse (10-12). The experimental approaches developed were used to tackle issues related to abnormal chromosome disjunction during meiosis, and showed murine metaphase II spindles, with complementary information on chromosome position. Furthermore, observations in human metaphase II spindles have also been briefly described (5). It should be noted that cytoplasmic interference is not usually an issue in these experiments, given that the cytoplasm has either been removed (chromosome spreads) or that the DNA has a peripheral, cortical, localization relative to the oocyte (metaphase II spindles, polar bodies).

Simultaneous evaluation of human oocyte structures and proteins by immunofluorescence is straightforward, provided the individual protocols involve similar methodologies (13). The standard procedure, adapted to individual settings, calls for formaldehyde/paraformaldehyde or methanol fixation, followed by Triton X-100 permeabilization/wash, and incubation of samples with the appropriate probes. However, as we describe, the most common protocols for ICC and FISH are not very similar and can interfere with one another, rendering some samples useless for analysis in the process.

Therefore, we report a simple method that can be employed to analyze not only metaphase II spindles but also human failed fertilizations arrested at several stages of development using, in this case, both tubulin ICC for microtubular patterns and FISH for specific chromosome positioning. The technique preserves the sample morphology ascertained by ICC, even after the harsher conditions normally required for probe hybridization during FISH.

\section{MATERIALS AND METHODS}

All chemicals were obtained from Sigma Chemical Company (St. Louis, MO) unless stated otherwise. All human material was used in accordance with the appropriate ethics guidelines provided by the University Hospitals of Coimbra.

\section{Biologic Material}

The human fertilization failures analyzed were obtained from the fertility clinic (University Hospitals of Coimbra) from consenting patients undergoing fertility treatments involving both IVF and ICSI, following appropriate institutional review board guidelines. Failed fertilized oocytes were analyzed that displayed signs of activation (second polar body extrusion) without pronuclear development or had an absence of polar body emission or cleavage 48 hours after insemination or microinjection. A total of 161 oocytes/failed human fertilizations were available for this study.

\section{Tubulin Immunocytochemistry and Fluorescence in Situ Hybridization}

The zona pellucida was removed with a short (1 to 5 minutes) incubation in acid Tyrode's solution. Oocytes were fixed for 1 hour with $2 \%(v / v)$ formaldehyde in Ferticult medium (FertiPro N.V., Beernem, Belgium) and permeabilized in 5\% Triton X-100 $(v / v)$ in phosphate-buffered saline (PBS; pH 7.2) for 1 to 2 hours at room temperature. Nonspecific antibody reactions were blocked by a 30-minute incubation in PBS containing $1 \mathrm{mg} / \mathrm{mL}$ of bovine serum albumin and $100 \mathrm{mM}$ of glycine.

For labeling, sheep anti-human tubulin polyclonal antibody (Cytoskeleton, Denver, CO) was solubilized in the blocking solution (1:200 dilution) and incubated with the samples for 1 hour at $37^{\circ} \mathrm{C}$. After washing in PBS containing $0.1 \%$ Triton X-100 $(v / v)$, the oocytes were labeled with Alexa Fluor 488 or Alexa Fluor 568 donkey anti-sheep IgG (Molecular Probes, Eugene, OR) at a 1:200 dilution in blocking solution for 40 to 60 minutes at $37^{\circ} \mathrm{C}$, then washed again with PBS containing $0.1 \%$ Triton X-100. To stain the DNA, each oocyte was put in a slide with a $10-\mu \mathrm{L}$ drop of antifade solution with $0.1 \mu \mathrm{g} / \mathrm{mL}$ of 4,6-diamidino-2-phenylindoleDAPI (Appligene, Tucson, AZ). The slides were then covered with coverslips and sealed with nail polish. Oocytes were examined with a Zeiss Axiophot II microscope equipped with a triple band pass filter.

After ICC analysis and photography, the coverslips were then carefully removed and the oocytes, which stayed attached to the slides, were gently rinsed with $0.1 \%$ Triton $\mathrm{X}-100$ in PBS and prepared for FISH. The DNA probes (Vysis, Inc., Downers Grove, IL) used for this study were centromeric fluorescent probes for chromosomes X, Y, and 18 (CEP X Spectrum Green, CEP Y Spectrum Orange, and CEP 18 Spectrum Orange), applied both individually and in combination. Fixation with acetic acid/methanol or an ethanol dehydration series were not used.

The FISH procedure was performed according to the protocol recommended by the manufacturer for directly labeled probes: $10 \mu \mathrm{L}$ of the probe mixture was applied to each slide under a coverslip sealed with rubber cement. The DNA was denatured at $75^{\circ} \mathrm{C}$ on a hot plate for 5 minutes and hybridization took place overnight in a humidified chamber 
at $42^{\circ} \mathrm{C}$. Coverslips were removed, and the slides were washed twice: first, in $0.4 \times \mathrm{SSC}$ solution with $0.3 \%$ Igepal (Appligene) at $75^{\circ} \mathrm{C}$ for 1 minute, then, with $2 \times \mathrm{SSC} / 0.1 \%$ Igepal solution for 1 minute at room temperature. Slides were mounted in antifade medium containing $0.1 \mu \mathrm{g} / \mathrm{mL}$ of DAPI (Appligene) and analyzed with a Zeiss Axiophot II microscope as previously noted.

\section{RESULTS}

Initial attempts to first analyze and document tubulin ICC and then perform FISH on the same sample using the respective standard methodologies were largely unsuccessful due to several factors. Some steps normally required for FISH (ethanol/acetic acid fixation, ethanol dehydration) seemed to disrupt the chromatin structure or erase the microtubular staining patterns detected by ICC (data not shown). On the other hand, standard permeabilization of samples in 1\% Triton X-100 for 30 minutes following formaldehyde fixation, although suitable for ICC, rendered the samples opaque following the heating steps required for FISH probe hybridization, probably due to massive cytoplasmic protein denaturation (data not shown). As a consequence, only chromatin at the cell surface could be unequivocally labeled with FISH probes, namely, metaphase II plates and polar bodies as already described (7, 10-12).

We tackled these issues with two methodological shifts. To avoid oocyte opacity following FISH analysis, we increased the permeabilization used for ICC. Following fixation with formaldehyde, samples were thus permeabilized for 1 to 2 hours with 5\% Triton X-100 in PBS. This was particularly effective in removing enough of the oocyte cytoplasm so that the heating steps necessary for FISH did not render the cell opaque. On the other hand, we found that, after ICC data had been collected for a given sample, it was no longer necessary to perform further fixation steps for FISH analysis with methanol/ acetic acid or proceed through the ethanol dehydration series (data not shown).

Figure 1A shows an oocyte that failed to properly activate following IVF, in which chromosomal misalignments at the metaphase II spindle are obvious (see also 7, 10-12). The spindle characteristics and microtubular staining were maintained after FISH analysis, as carried out with probes for chromosomes 18 and X added simultaneously (see Fig. 1A', $\mathrm{A}^{\prime \prime}$ ). Two adjacent FISH spots are visible for both probes, indicating the presence of two chromatids. Note that, even if microtubules and a FISH probe must be visualized using the same emission channel (see Fig. 1A"), differences between the types of signals (linear or twisted microtubular patterns vs. clear FISH dots) should be enough to provide for adequate interpretation of the data.

This can also be seen in Figure 1B, in which failure of the oocyte to fertilize was followed by rescue ICSI 20 hours

\section{FIGURE 1}

Chromosome positioning can be assessed in human failed fertilizations without compromising cytoskeletal patterns. (A) Human failed fertilization with misaligned chromosomes visualized first by tubulin immunocytochemistry (green) and then using FISH for both chromosome 18 (red spots, $A^{\prime}$ ) and $\mathrm{X}$ (green spots, $\left.A^{\prime \prime}\right)$. (B) An arrested human fertilization was sequentially probed with the antitubulin antibody (green) and with FISH probes for X (green) and 18 (red). Three sets of chromosomes were visible. Insert $B^{\prime}$ is a detail in which the microtubule pattern has been removed for clarity. (C) An arrested human fertilization was sequentially probed with the antitubulin antibody (green) and with FISH probes $\left(C^{\prime}\right)$ for $X$ (green) and $18(\mathrm{red})$. Asterisks map the localization of the indentation fossae, noting the putative position of the maleborne centrosome. DNA is in blue. Bars represent $10 \mu \mathrm{m}$.
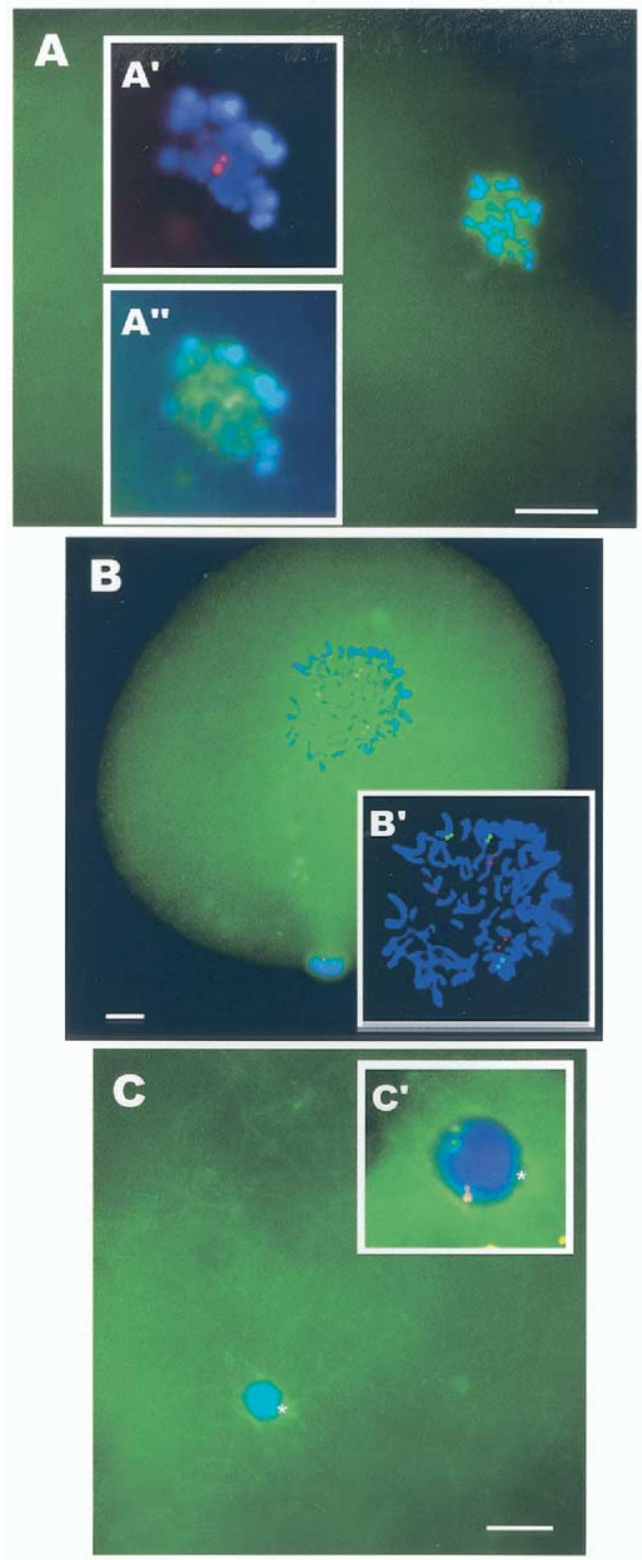

Ramalho-Santos. ICG and FISH in human fertilization failures. Fertil Steril 2004. 


\section{FIGURE 2}

Human failed fertilizations at the onset of first mitosis can be analyzed by both microtubular staining and FISH. Human fertilization failures were sequentially probed with the antitubulin antibody (red) and with FISH probes. (A) View of a fertilized human zygote arrested at anaphase of first mitosis stained for microtubules (red) and DNA (blue). A $A^{\prime}$ : Detail of $A$, showing the mitotic apparatus. $A^{\prime \prime}$ : The same zygote following FISH analysis using probes for chromosomes X (green spots), Y (large red spots), and 18 (small red spots). Note the presence of the sperm tail. (B) A parthogenetic human oocyte, arrested at telophase of first mitosis, stained for microtubules (red) and DNA (blue). B': The same cell following FISH analysis with probes for chromosomes $\mathrm{X}$ (green spots) and 18 (small red spots). A probe for chromosome $\mathrm{Y}$ was also used, but no signal was detected (not shown). Note the presence of the midbody. Bars represent $10 \mu \mathrm{m}$.
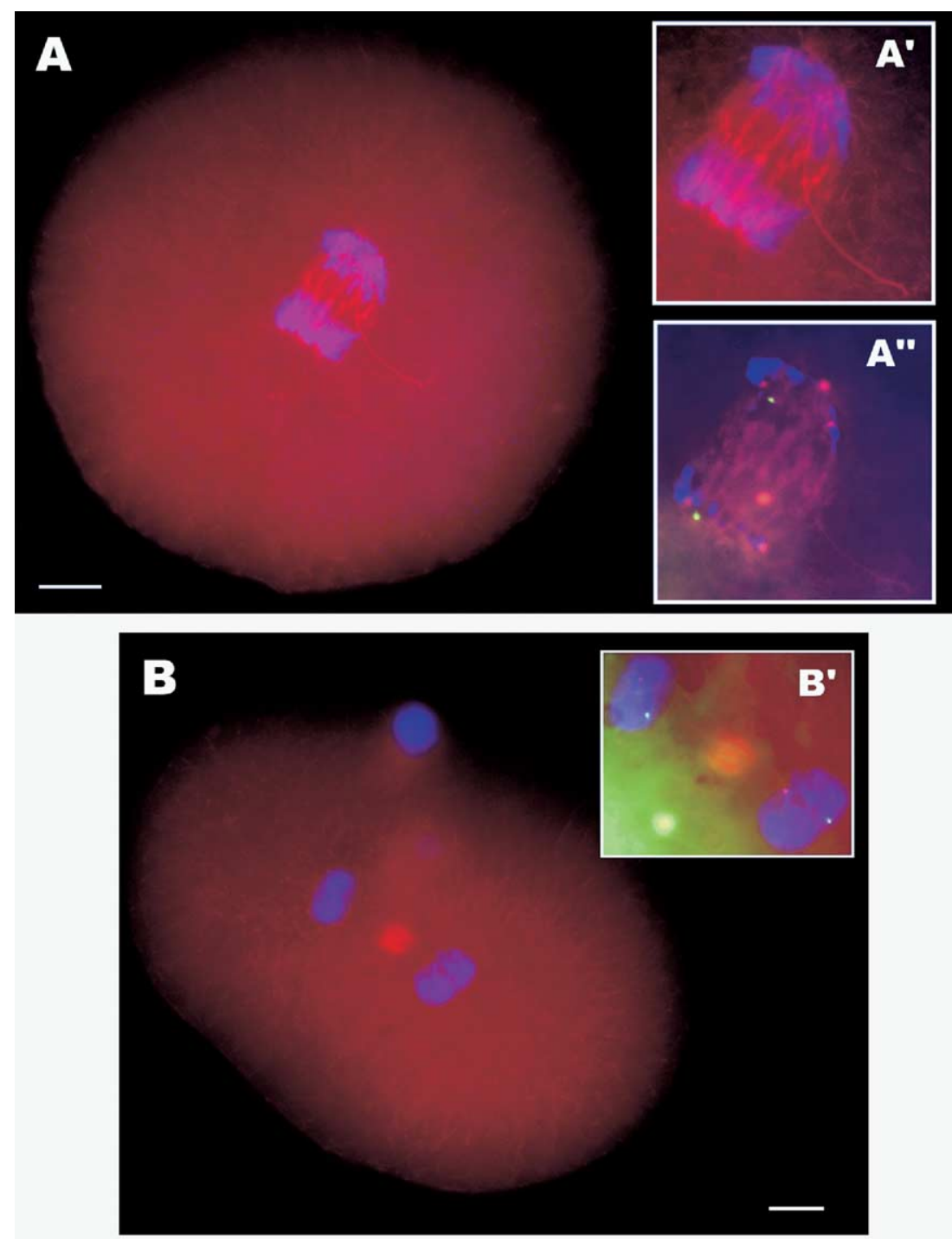

Ramalho-Santos. ICG and FISH in human fertilization failures. Fertil Steril 2004.

after conventional insemination. The oocyte was then diagnosed in the clinic as a 3PN (possible retention of the second polar body) and made available for analysis. Analysis by ICC-FISH revealed three sets of condensed chromosomes with a very loose microtubule network that failed to organize a spindle.

More importantly, the technique could also be applied to pronuclear stage embryos. Figure $1 \mathrm{C}$ shows a failed human fertilization following ICSI in which the male pronucleus, although partially decondensed, not only can be identified by morphologic features, but also has failed to properly organize a microtubular aster. This disorganized microtubule pattern arising from the would-be centrosome area suggests that this was probably the cause for fertilization arrest. Interestingly, the dual spots for chromosomes $\mathrm{X}$ and 18 revealed by FISH analysis (see Fig. $1 \mathrm{C}^{\prime}$ ) suggest that DNA 
FIGURE 3

Human failed fertilizations after first cleavage analyzed by both microtubular staining and FISH. A human embryo that arrested at the two-cell stage was sequentially probed with immunocytochemistry and with FISH. (A) ICC: Staining using the antitubulin antibody (red) and the DNA stain DAPI (blue). (B) The same embryo following FISH analysis using probes for chromosomes X (green spots) and 18 (red spots). DNA is in blue. Bars represent $10 \mu \mathrm{m}$.
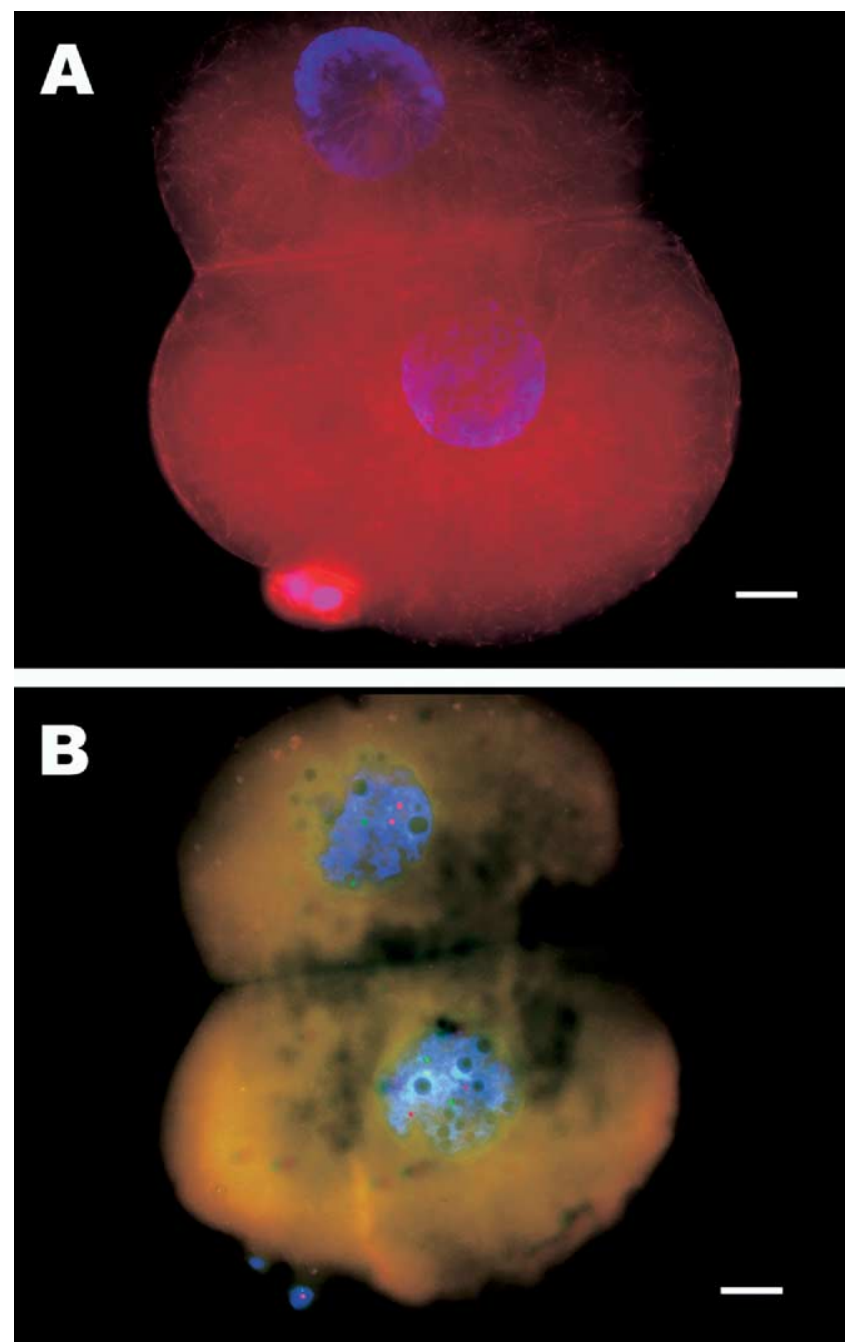

Ramalho-Santos. ICG and FISH in human fertilization failures. Fertil Steril 2004.

synthesis had already begun, similar to what has been described for other primate species following cytoplasmic removal of sperm structures after ICSI (14) but before pronuclear apposition.

We were also able to analyze human fertilization failures that failed to progress beyond the first mitotic division. Figure 2 shows two failed fertilizations from the same 33year-old patient with endometriosis undergoing IVF. The first embryo analyzed had arrested at anaphase of first mitosis (see Fig. 2A). With this specimen, we were also able to show that more than one FISH probe can be used in the same fluorescence channel, provided the signals are sufficiently different as is the case with the probes for chromosomes 18 (small red dots) and Y (large red dots) used here (see Fig. $\left.2 \mathrm{~A}^{\prime \prime}\right)$. Note that chromosome $\mathrm{Y}$ does not seem to have segregated properly, and that the sperm tail is clearly visible, both of which confirm that fertilization took place.

The same analysis was carried out in another failed fertilization from the same patient, in which activation was not observed. However, the embryo was found to have arrested at telophase of first mitosis (see Fig. 2B). In this case, the individual signals obtained with probes for chromosomes 18 and $\mathrm{X}$ in each chromatin (the $\mathrm{Y}$ chromosome was not detected) suggest that this failed fertilization may have resulted from parthenogenetic activation (see Fig. 2B').

Finally, Figure 3 shows a failed human fertilization that arrested as a two-cell embryo. Signs of proper oocyte activation are visible in the network of cortical microtubules revealed by ICC (see Fig. 3A). Unlike sturdier microtubular structures, such as spindles (see Fig. 1A), the sperm aster (see Fig. 1C), the sperm tail (see Fig. 2A"), or the midbody (see Fig. 2B'), these more delicate cortical microtubules were disrupted following FISH analysis (see Fig. 3B). However, the morphologic features of the general embryo (note especially the nucleolar structure) were maintained, and FISH analysis was carried out successfully; two distinct spots for chromosome 18 and the $\mathrm{X}$ chromosome were clearly visible in both nuclei, and additional signals also were detected in the polar body (see Fig. 3B). Note the slight opacity induced in the arrested zygote after FISH analysis, probably due to the aforementioned cytoplasmic protein denaturation. In similar samples that had only been permeabilized with a short incubation in $1 \%$ Triton X-100, FISH analysis was not possible unless the DNA was close to the cell surface, as previously described.

\section{DISCUSSION}

The parallel use of ICC and FISH analysis in relation to human fertility has already been demonstrated, notably in cytogenetic analysis which also is interested in investigating the localization of specific proteins on chromosomes (7-9). These observations are usually done at the chromosome level with prior removal of cell cytoplasm. Further work has been carried out on metaphase II plates to study chromosomal disjunction in the mouse $(7,10-12)$ but also with some human data (5). We have described a simple technique that allows the parallel analysis of microtubular patterns using tubulin ICC and chromosome positioning/status using FISH in the same samples of failed human fertilizations, which were arrested not only at the metaphase II stage but at distinct stages of development. This approach was used because defects in the proper organization of microtubular patterns are well known for arresting human fertilization at distinct points, depending on the deficiency observed in each case (2). 
The methodology may be useful for example, in determining which specific chromosomes are missorted or misaligned in oocytes or zygotes from specific patients, in assessing whether arrest took place before or after activation of DNA synthesis in the pronuclei, or in distinguishing failed fertilization from failed parthenogenetic activation. More importantly the optimized protocol retains the structural morphology of the sample without gross distortions and can be used regardless of chromatin position in the oocyte; that is, the DNA does not need to be close to the cell surface.

Simultaneous detection of several types of molecules, organelles, or structures in a human oocyte or any other living cell is straightforward (13), provided that several conditions are fulfilled. For example, the appropriate probes must be available, and they must not overlap in specificity or in the emitted signals. The final hurdle is determining if the methodology used for different probes/ analysis is compatible. For example, some probes can only be used to label live cells, while others work best with fixed material. In this particular case, the most challenging aspect was avoiding cytoplasmic interference, which would render the samples opaque after FISH. This issue was resolved using increased detergent-mediated permeabilization. It is therefore important to note that applying this technique to compare the localization of particular proteins/cellular structures may be limited by the nature of the protein/structure in question. If it is lost or altered following extensive permeabilization, for example, the results may be inconclusive or may not reflect the accurate localization/concentration of a given molecule of interest. This is particularly important in the analysis of human oocytes or arrested zygotes, given that cell size and the abundance of concentrated cytoplasm can interfere with analysis if they are not adequately processed. A balance must be struck between the two (or more) methodologic approaches that need to be applied, simultaneously or sequentially. Therefore, the protocol must be optimized for each particular analysis, with care being taken that a given sample is not irreversibly dis- rupted at any stage, thus rendering direct comparisons between techniques difficult.

Acknowledgments: The authors thank Dr. Ana Barbosa, Conceição Dias, and Paula Henriques (University Hospitals of Coimbra) for many helpful discussions and technical assistance, and Prof. Agostinho Almeida Santos (University Hospitals of Coimbra) for continuous encouragement and support. We also thank Dr. H. Beard for thoughtful advice.

\section{References}

1. Trounson AO, Gosden RG, eds. Biology and pathology of the oocyte: its role in fertility and reproductive medicine Cambridge, UK: Cambridge University Press, 2003.

2. Asch R, Simerly C, Ord T, Ord VA, Schatten G. The stages at which human fertilization arrests: microtubule and chromosome configurations in inseminated oocytes which failed to complete fertilization and development in humans. Hum Reprod 1995;10:1897-906.

3. Coonen E, Hopman AHN, Geraedts JPM, Remaekers CS. Application of in-situ hybridization techniques to study human preimplantation embryos: a review. Hum Reprod Update 1998;4:135-52.

4. Schatten G. The centrosome and its mode of inheritance: the reduction of the centrosome during gametogenesis and its restoration during fertilization. Dev Biol 1994;165:299-335.

5. Hassold T, Hunt P. To err (meiotically) is human: the genesis of human aneuploidy. Nat Rev Genet 2001;2:280-91.

6. Barlow AL, Tease C, Hulten MA. Meiotic chromosome pairing in fetal oocytes of trisomy 21 human females. Cytogenet Genome Res 2002; 96:45-51.

7. Hodges CA, LeMaire-Adkins R, Hunt PA. Coordinating the segregation of sister chromatids during the first meiotic division: evidence for sexual dimorphism. J Cell Sci 2001;114:2417-26.

8. Hodges CA, Hunt PA. Simultaneous analysis of chromosomes and chromosome-associated proteins in mammalian oocytes and embryos. Chromosoma 2002;111:165-9.

9. Tease C, Hartshorne GM, Hulten MA. Patterns of meiotic recombination in human fetal oocytes. Am J Hum Genet 2002;70:1469-79.

10. Hunt P, LeMaire R, Embury P, Sheean L, Mroz K. Analysis of chromosome behavior in intact mammalian oocytes: monitoring the segregation of a univalent chromosome during female meiosis. Hum Mol Genet 1995;4:2007-12.

11. LeMaire-Adkins R, Radke K, Hunt PA. Lack of checkpoint control at the metaphase/anaphase transition: a mechanism of meiotic nondisjunction in mammalian females. J Cell Biol 1997;139:1611-9.

12. LeMaire-Adkins R, Hunt PA. Nonrandom segregation of the mouse univalent $\mathrm{X}$ chromosome: evidence of spindle-mediated meiotic drive. Genetics 2000;156:775-83.

13. Simerly C, Moreno RD, Ramalho-Santos J, Hewitson L, Schatten G. Confocal imaging of structural molecules in mammalian gametes. In: Gardner DK, Lane M, eds. A laboratory guide of the mammalian embryo. Oxford, UK: Oxford University Press, 2003.

14. Ramalho-Santos J, Sutovsky P, Simerly C, Oko R, Wessel G, Hewitson L, et al. ICSI choreography: fate of sperm structures after monospermic rhesus ICSI and first cell cycle implications. Hum Reprod 2000;15: $2610-20$. 